\title{
Relationship duration and mental health outcomes: findings from a 30-year longitudinal study
}

\author{
Sheree J. Gibb, David M. Fergusson and L. John Horwood
}

\section{Background}

Marriage is known to be associated with improved mental health, but little research has examined whether the duration of a cohabiting relationship is associated with mental health.
Aims
To examine the associations between relationship duration and mental health problems in a birth cohort of 30-year-olds.

\section{Method}
Associations between relationship duration and mental health were examined using a generalised estimating equation approach. Associations were adjusted for covariates, including prior mental health problems.

\section{Results}

Longer relationship duration was significantly associated with lower rates of depression, suicidal behaviour and substance abuse/dependence, even after adjustment for covariates. In most cases the associations did not vary with gender. Legal relationship status (legally or de facto married) was not significantly related to mental health once due allowance was made for relationship duration.

\section{Conclusions}

Increasing relationship duration, but not legal relationship status, has a protective effect on mental health for men and women.

\section{Declaration of interest}

None.
Previous research has suggested that, compared with unmarried individuals, married individuals have lower rates of depression, anxiety and substance use, and have higher levels of well-being and life satisfaction. ${ }^{1-6}$ It has been suggested that the associations between marriage and mental health may reflect selection effects, with psychologically healthy individuals more likely to become married. ${ }^{4,5,7}$ However, controlling for prior mental disorder and other confounding factors reduces, but does not eliminate, the associations between marriage and mental disorder. ${ }^{4,7}$ Although early research suggested that marriage had greater benefits for males than for females, ${ }^{8}$ more recent studies suggest that marriage has similar benefits for both genders. ${ }^{1-3,9}$

A limitation of existing research is that it has focused largely on legal marriage and has paid less attention to the role of nonmarriage cohabiting relationships. The small number of studies that have examined non-marriage cohabiting relationships have reported that cohabitation has smaller or no effects on rates of mental disorder. ${ }^{2,7,9}$ However, this conclusion may be misleading since, on average, cohabiting relationships may be of shorter duration than legal marriages. This may suggest that the critical dimension of a relationship that influences mental health is not the legal status of the relationship but rather its duration. It may be hypothesised that when due allowance is made for the duration of a relationship the legal status of the union is unrelated to rates of mental disorder.

Against this background, this paper presents the results of a longitudinal study of the associations between relationship duration and mental disorder in a cohort of New Zealand-born children studied to the age of 30 .

The specific aims of the study were:

(a) to examine the associations between relationship duration and mental disorder;

(b) to adjust the associations between relationship duration and mental disorder for covariate factors, including prior mental disorder; (c) to examine whether the legal status of a relationship is significantly associated with mental disorder once due allowance has been made for relationship duration;

(d) to examine whether the adjusted associations between relationship duration and mental disorder are different for men and women.

\section{Method}

\section{Participants}

Data were collected as part of the Christchurch Health and Development Study, a longitudinal study of a birth cohort of 1265 individuals born in Christchurch, New Zealand, in 1977 and followed to age 30. Participants were followed up at birth, 4 months, 1 year, yearly to age 16 , then again at ages 18 , 21, 25 and 30. Data were collected using a combination of semistructured interviews, standardised testing and teacher reports, on the basis of signed consent from study participants. The major findings of this study have been reviewed previously. ${ }^{10,11}$

\section{Measures}

Relationship duration

At the age 25 and 30 interviews, participants were questioned about their partner relationships over the preceding 12 months. Participants who reported being involved in a cohabiting relationship during the interval were asked to report the total length of that relationship. Relationship duration was classified into four categories: no relationship $(44.8 \%$ of participants at age $24-25$ and $28.0 \%$ at age $29-30$ ); relationship of less than 2 years $(16.5 \%$ of participants at age $24-25$ and $20.5 \%$ at age 29-30); relationship of 2 to 4 years $(22.6 \%$ of participants at age $24-25$ and $20.4 \%$ at age $29-30$ ); and relationship of 5 or more years $(16.2 \%$ of participants at age $24-25$ and $31.2 \%$ at age 29-30). 


\section{Legal status of relationship}

At age 24-25 and age 29-30, participants were classified into three groups according to the legal status of their cohabiting relationship: no relationship; de facto married (cohabiting but not legally married); or legally married. Participants who were involved in a de facto marriage (known as a de facto relationship in New Zealand) that became a legal marriage over the interval were classified as legally married. At age $24-25,44.8 \%$ of participants were not in a relationship, $45.1 \%$ were in a de facto relationship, and $10.2 \%$ were legally married. At age 29-30, $28.0 \%$ were not in a relationship, $43.9 \%$ were in a de facto relationship and $28.1 \%$ were legally married.

For the purposes of the regression models, legal status of relationship was represented by two design variates specifying whether or not participants were in a de facto relationship, and whether or not participants were in a legal marriage.

\section{Psychiatric disorder}

At ages 25 and 30, participants were questioned about the extent to which they had suffered from symptoms of depression, generalised anxiety disorder, panic disorder, social phobia, specific phobias, agoraphobia, and substance abuse and dependence in the 12 months preceding the interview. Questions were drawn from the Composite International Diagnostic Interview. ${ }^{12}$ Responses to these questions were used to determine the presence or absence of a series of psychiatric disorders based on DSM-IV ${ }^{13}$ criteria (see Fergusson et al $^{14}$ for a more detailed explanation of the criteria used). In addition, participants were questioned using customwritten survey items about the occurrence of suicidal thoughts or suicide attempts over the preceding 12 months. Responses to the above questions were used to construct the following measures.

Depression. This measure represented the presence or absence of a diagnosis of major depression during the interval. Overall, $14.6 \%$ of participants met criteria for depression at age 24-25, and $13.6 \%$ at age $29-30$.

Anxiety disorder. This measure represented the presence or absence of any anxiety disorder diagnosis (generalised anxiety disorder, panic disorder, social phobia, agoraphobia or specific phobia) during the interval. Overall, $13.2 \%$ of participants met criteria for anxiety disorder at age $24-25$, and $14.7 \%$ at age $29-30$.

Suicidal ideation/attempt. This measure represented whether or not participants reported either suicidal ideation (contemplating, considering or planning suicide) or suicide attempt during the interval. Overall, suicidal ideation/attempt was reported by $6.5 \%$ of participants at age $24-25$ and $4.6 \%$ at age $29-30$.

Alcohol abuse/dependence. This measure represented the presence or absence of a diagnosis of either alcohol abuse or alcohol dependence during the interval. Overall, $13.6 \%$ of participants met criteria for alcohol abuse/dependence at age $24-25$, and $9.0 \%$ at age $29-30$.

Illicit drug abuse/dependence. This measure represented the presence or absence of a diagnosis of cannabis or other illicit drug abuse or dependence during the interval. Overall, $10.7 \%$ of participants met criteria for illicit drug abuse/dependence at age $24-25$, and $5.1 \%$ at age $29-30$.
Total number of psychiatric disorders. A measure of the overall burden of mental health problems was based on a count of the number of mental health problems reported by the participant at each age. This measure was the sum of the five dichotomous mental health problem measures described above. At age $24-25,62.7 \%$ had no mental health problems, $22.7 \%$ had one, $9.7 \%$ had two, and $4.9 \%$ had three or more problems. At age $29-30,68.3 \%$ had no mental health problems, $20.9 \%$ had one, $7.4 \%$ had two, and $3.4 \%$ had three or more problems.

\section{Gender}

Gender was the participant's sex reported at the birth interview. At age $25,48.7 \%$ of the sample was male, and at age $30,48.4 \%$ was male.

\section{Covariate factors}

To control the association between relationship duration and mental health for confounding, a range of potential covariate measures were selected from the study database. These included measures of: family background (parental history of offending, illicit drug use and alcohol abuse; maternal age; maternal education); childhood family functioning (family socioeconomic status at birth; parental changes to age 15; family living standards age 0-10; exposure to childhood adversity to age 15); child abuse (sexual abuse and physical punishment before age 16); cognitive ability and academic achievement (total IQ at age 8/9; age left secondary school; Test of Scholastic Abilities ${ }^{15}$ score at age 13; teacher-rated academic performance at age 11-13); individual characteristics and behaviours (neuroticism at age 14; noveltyseeking at age 16; deviant peer affiliations at age 16; mother-rated conduct problems at age 16; ethnicity; early sexual intercourse, number of dependent children); prior mental health problems (depression, anxiety, suicidal ideation/attempt, alcohol abuse/ dependence, illicit drug abuse/dependence in the preceding interview period); prior and current relationships (number of previous cohabiting relationships, relationship separation during previous interview period); recent adverse life events (employment problems; death of family/friend; family arguments; serious illness of self or family/friend; being a victim of burglary, robbery or assault).

These measures were refined on the basis of the regression analyses to a subset of variables that were significant $(P<0.05)$ in at least one analysis. Those variables are described below.

Parental history of illicit drug use. When participants were aged 11 , their parents were questioned about their use of cannabis and other illicit drugs. On the basis of this questioning, a variable was constructed representing whether or not participants' parents reported having ever used illicit drugs.

Family socioeconomic status. The socioeconomic status of the participant's family was measured at birth using the Elley-Irving scale of socioeconomic status, ${ }^{16}$ which classifies socioeconomic status on a six-level scale according to paternal occupation. For simplicity, this scale was condensed to three levels where 1 was professional/managerial, 2 was clerical/technical/skilled and 3 was semi-skilled/unskilled/unemployed.

Exposure to childhood adversity. This variable was a count measure of 39 measures of family disadvantage during the period 0-15 years, including measures of disadvantaged parental background, poor prenatal health practices and perinatal outcomes, and disadvantageous child-rearing practices. ${ }^{17}$ 
IQ. At ages 8 and 9, participants were assessed with the Revised Wechsler Intelligence Scale for Children. ${ }^{18}$ The measure used in the present analysis was the average of the total IQ scores at ages 8 and 9 and had a reliability (split-half) of 0.95 .

Age left secondary school. Information from interviews at ages 16,18 and 21 was used to determine the age, in years, at which participants left secondary school.

Test of Scholastic Abilities. At age 13, participants completed the Test of Scholastic Abilities, ${ }^{15}$ which measures whether students possess the verbal and numerical reasoning abilities required for high-school success. This measure had a reliability of $\alpha=0.95$.

Teacher-rated academic performance. At ages 11, 12 and 13, participants' school teachers were asked to rate the participant's performance in reading, handwriting, written expression, spelling and mathematics using a five-point scale ranging from very good to very poor. Ratings were averaged across years and curriculum areas to provide a global measure of academic performance. This measure had a reliability of $\alpha=0.96$.

Mother-rated conduct problems. When participants were aged 16 , parents were questioned about the young person's behaviour using the Revised Behavior Problem Checklist. ${ }^{19}$ Selected items from these data were summed to provide a measure of conduct problems at age 16. This measure had a reliability of $\alpha=0.86$.

Neuroticism. At age 14, participants completed the short form version of the neuroticism scale of the Eysenck Personality Inventory. ${ }^{20}$ This measure had a reliability of $\alpha=0.80$.

Novelty-seeking. Novelty-seeking was assessed using the Tridimensional Personality Questionnaire ${ }^{21}$ administered when participants were aged 16 years. This measure had a reliability of $\alpha=0.76$.

Deviant peer affiliations. This measure represented the extent to which a participant reported at age 15 that their friends were involved in deviant behaviours, including nicotine, alcohol and illicit drug use, violent and property offending, and truancy and suspension from school. ${ }^{22}$

Childhood physical punishment. At ages 18 and 21, participants were questioned about the extent to which their parents had used physical punishment during the participant's childhood (before age 16). This information was used to construct a four-level scale representing the most extreme form of childhood physical punishment reported at either age 18 or 21: parents never used physical punishment $(4.5 \%$ of sample); parents rarely used physical punishment $(77.9 \%)$; at least one parent used physical punishment regularly $(11.2 \%)$; and at least one parent used physical punishment too often or severely, or treated the participant in a harsh or abusive manner $(6.4 \%) .^{23}$

Childhood sexual abuse. At ages 18 and 21, participants were questioned about their exposure to sexual abuse during childhood (before age 16). This information was used to construct a fourlevel scale representing the most extreme form of childhood sexual abuse reported by the participant at either age 18 or 21: no abuse (85.9\% of sample); non-contact abuse only (2.7\%); contact abuse not involving attempted or completed intercourse (5.1\%); and attempted/completed oral, anal or vaginal intercourse $(6.3 \%) .{ }^{14}$
Number of previous cohabiting relationships. This measure was the total number of cohabiting relationships (excluding current relationship) that participants had been in by age 25 and age 30 .

Prior psychiatric disorder. Prior psychiatric disorder during the preceding interview period (age 18-21 or age 21-25) was measured by five dichotomous variables representing the presence or absence of: major depression, anxiety disorder, suicidal ideation/attempt, alcohol abuse/dependence and illicit drug abuse/dependence. These variables were calculated in the same way as the psychiatric disorder variables described previously.

\section{Statistical methods}

The associations between the repeated measures of relationship duration and mental disorder were tested for statistical significance by fitting regression models to the data for each mental disorder outcome using a generalised estimating equation (GEE) approach. ${ }^{24,25}$ The GEE approach pooled the repeated measures on each relationship duration measure and on each mental health outcome at ages 24-25 and 29-30 years to produce an estimate of the population-averaged association between relationship duration and the mental health outcome. For dichotomous mental health measures, a logistic regression model was fitted of the form:

$$
\operatorname{logit}\left(Y_{i t}\right)=B_{0}+B_{1} X_{i t}
$$

where $\operatorname{logit}\left(Y_{i t}\right)$ was the $\log$ odds of the outcome $Y$ for the $i$-th participant in the $t$-th time period ( $t=24-25,29-30$ years), and $X_{i t}$ was relationship duration for the $i$-th individual in the $t$-th time period. For the count of the total number of mental disorders, a Poisson regression model was fitted of the form:

$$
\log \left(Y_{i t}\right)=B_{0}+B_{1} X_{i t}
$$

where $\log \left(Y_{i t}\right)$ was the logarithm of the rate of disorder for the $i$-th individual in the $t$-th time interval. In all cases the fitted model permitted the repeated measures of the outcomes to be correlated.

In these models the coefficient $B_{1}$ represents the effect of relationship duration on the mental health outcome pooled over the two observation periods. In each case the fitted model assumed a linear effect of relationship duration, and a test of significance of the association was given by a Wald chi-squared test of the hypothesis that $B_{1}=0$.

To adjust the observed associations between relationship duration and the mental health outcome measures for confounding factors, the GEE models above were extended to include the set of covariate factors listed in the Method section above. All covariate factors were initially included, and then the model was successively refined to remove those that were not significant $(P<0.05)$ predictors of the outcome. For dichotomous outcomes the model fitted was of the form:

$$
\operatorname{logit}\left(Y_{i t}\right)=B_{0}+B_{1} X_{i t}+\Sigma B_{j} Z_{i j}+\Sigma B_{k} Z_{i k t}
$$

and for the count of number of mental health problems the model was of the form:

$$
\log \left(Y_{i t}\right)=B_{0}+B_{1} X_{i t}+\Sigma B_{j} Z_{i j}+\Sigma B_{k} Z_{i k t}
$$

where $Z_{i j}$ were a set of fixed covariates and $Z_{i k t}$ a set of time dynamic covariate factors for individual $i$. In these models the coefficient $B_{1}$ represents the effect of relationship duration on 
the outcome net of the correlated effects of the other covariates. To examine the adequacy of the linearity assumption for the effect of relationship duration, the final fitted models for each outcome were extended by incorporating additional design variates representing categories of relationship duration to test for departures from linearity. Finally, to test the equivalence of the effect of relationship duration on each outcome for men and women, the fitted regression models were extended so that the effect of relationship duration was nested within gender. The equality of the coefficients $B_{1}$ for men and women was then tested using a Wald chi-squared test. Adjusted proportions were calculated using the methods described by Lee. ${ }^{26}$ All statistical analyses were conducted in SAS 9.1 for Windows.

\section{Sample size and sample bias}

Over the course of the study there has been a gradual loss of participants due to participant refusal, death and inability to trace participants. The present analyses are based on the samples assessed at age 25 and 30. These sample sizes varied from 1003 at age 25 to 987 at age 30, representing between $81 \%$ and $80 \%$ of the surviving cohort at each age. By age 30, 34 participants $(2.7 \%)$ had died, $114(9.0 \%)$ had refused participation, and 130 $(10.3 \%)$ could not be traced.

The gradual loss of participants over the course of the study raises questions about the extent to which the results of this paper may be influenced by sample bias due to non-random sample loss. To examine this, missing data were imputed using the 'PROC MI' and 'PROC MIANALYZE' procedures in SAS 9.1 for Windows, which use multiple imputation to impute missing data and then use these data in the analysis to adjust standard error estimates under the assumption that there was no bias in the estimation of the missing data.

The major conclusions of the analysis using the imputed data were the same as those obtained using the original sample, suggesting that the results of this paper were not significantly affected by sample bias. Therefore, the remainder of this paper reports the results of the analyses of the original (non-imputed) data.

\section{Results}

\section{Unadjusted associations between relationship duration and mental disorders}

Table 1 shows the cohort classified according to the duration of partner relationship (none, up to 2 years, 2-4 years, or 5+ years) at ages 24-25 and 29-30. For each group, the table shows rates of selected mental disorders at ages 24-25 and 29-30 and pooled rates derived from a population-averaged GEE model (see Method for details). Table 1 shows that with the exception of anxiety disorders, increased duration of relationship was significantly $(P<0.0005)$ associated with declining rates of a range of mental health disorders, including depression, suicidal ideation/attempt, alcohol abuse/dependence, illicit drug abuse/dependence, and total number of mental health problems. The results suggest that those in a relationship of 5 years or longer had overall rates of mental disorder that were approximately $56 \%$ of the rates of those not in relationships.

\section{Adjusted associations between relationship duration and mental disorder}

Table 2 shows the pooled associations between duration of relationship and mental health problems adjusted for covariate factors, including measures of: family background; childhood family functioning; childhood abuse; cognitive ability and academic achievement; individual characteristics and behaviours; prior history of mental health problems; prior and current relationships; and recent adverse life events (see Method for details). For each mental health outcome, the table reports the adjusted regression coefficient $(B)$ and standard error (s.e.) for the relationship duration effect.

Table 1 Associations between relationship duration and mental disorder

\begin{tabular}{|c|c|c|c|c|c|}
\hline & \multicolumn{4}{|c|}{ Relationship duration, years } & \multirow[b]{2}{*}{$P$} \\
\hline & 0 & $<2$ & $2-4$ & $5+$ & \\
\hline \multicolumn{6}{|l|}{ Depression, \% } \\
\hline 24-25 years & 15.6 & 18.0 & 13.0 & 11.5 & \\
\hline 29-30 years & 15.6 & 23.0 & 9.8 & 9.2 & \\
\hline Overall & 15.6 & 20.8 & 11.5 & 10.0 & $<0.0005$ \\
\hline \multicolumn{6}{|c|}{ Anxiety disorder, \% } \\
\hline $24-25$ years & 12.9 & 11.5 & 13.0 & 15.0 & \\
\hline 29-30 years & 17.8 & 15.2 & 13.0 & 12.9 & \\
\hline Overall & 14.8 & 13.6 & 13.0 & 13.7 & $>0.79$ \\
\hline \multicolumn{6}{|c|}{ Suicidal ideation/attempt, \% } \\
\hline $24-25$ years & 7.4 & 7.9 & 7.4 & 2.5 & \\
\hline 29-30 years & 5.1 & 9.6 & 1.6 & 3.2 & \\
\hline Overall & 6.5 & 8.8 & 4.8 & 2.9 & $<0.0005$ \\
\hline \multicolumn{6}{|c|}{ Alcohol abuse/dependence, \% } \\
\hline 24-25 years & 18.5 & 18.7 & 6.5 & 6.5 & \\
\hline 29-30 years & 12.0 & 13.5 & 6.0 & 6.0 & \\
\hline Overall & 16.0 & 15.8 & 6.3 & 6.2 & $<0.0001$ \\
\hline \multicolumn{6}{|c|}{ Illicit drug abuse/dependence, \% } \\
\hline $24-25$ years & 13.1 & 15.8 & 7.9 & 4.5 & \\
\hline 29-30 years & 7.3 & 6.7 & 4.4 & 2.9 & \\
\hline Overall & 10.9 & 10.7 & 6.3 & 3.5 & $<0.0001$ \\
\hline \multicolumn{6}{|c|}{ Number of mental health problems, mean } \\
\hline $24-25$ years & 0.67 & 0.72 & 0.48 & 0.40 & \\
\hline 29-30 years & 0.58 & 0.68 & 0.35 & 0.34 & \\
\hline Overall & 0.64 & 0.70 & 0.42 & 0.36 & $<0.0001$ \\
\hline
\end{tabular}


Table 2 Covariate-adjusted ${ }^{a}$ associations between relationship duration and mental disorder

\begin{tabular}{|c|c|c|c|c|c|c|c|}
\hline & & & \multicolumn{4}{|c|}{ Legal status } & \multirow[b]{3}{*}{ Significant covariates ${ }^{a}$} \\
\hline & \multicolumn{2}{|c|}{ Relationship duration } & \multicolumn{2}{|c|}{ Marriage } & \multicolumn{2}{|c|}{ De facto relationship } & \\
\hline & $B$ & s.e. & $B$ & s.e. & $B$ & s.e. & \\
\hline Depression, \% & -0.19 & 0.06 & 0.70 & 0.39 & 0.63 & 0.28 & 12791013 \\
\hline Anxiety disorder, $\%$ & -0.02 & 0.07 & -0.31 & 0.42 & -0.20 & 0.34 & 127891519 \\
\hline Suicidal ideation/attempt, \% & -0.24 & 0.10 & 1.18 & 0.69 & 0.70 & 0.47 & 23671213 \\
\hline Alcohol abuse/dependence, \% & -0.35 & 0.08 & 0.44 & 0.50 & 0.13 & 0.32 & 34617 \\
\hline Illicit drug abuse/dependence, \% & -0.44 & 0.11 & -0.27 & 0.84 & 0.81 & 0.43 & 511141718 \\
\hline Number of mental health problems, mean & -0.19 & 0.03 & 0.02 & 0.20 & 0.14 & 0.13 & 12456710121316 \\
\hline \multicolumn{8}{|c|}{$\begin{array}{l}\text { a. Significant covariates: } 1 \text {, prior depression; } 2 \text {, prior anxiety disorder; } 3 \text {, prior suicidal ideation/attempt; } 4 \text {, prior alcohol abuse/dependence; } 5 \text {, prior illicit drug abuse/dependence; } 6 \text {, } \\
\text { number of prior cohabiting relationships; } 7 \text {, neuroticism at age } 14 ; 8 \text {, childhood adversity; } 9 \text {, contact sexual abuse at age }<16 ; 10 \text {, frequent or severe physical punishment at age } \\
<16 ; 11 \text {, teacher-rated academic progress at age } 11-13 ; 12 \text {, age left school; } 13 \text {, parental illicit drug use; } 14 \text {, Test of Scholastic Abilities }{ }^{15} \text { score at age } 13 ; 15 \text {, total IQ score at age } 8 / 9 \text {; } \\
\text { 16, novelty-seeking at age } 16 ; 17 \text {, deviant peer affiliations at age } 16 ; 18 \text {, maternal ratings of conduct problems at age } 16 ; 19 \text {, family socioeconomic status at birth. } \\
\text { b. Parameters in bold are statistically significant }(P<0.05) \text {. }\end{array}$} \\
\hline
\end{tabular}

Table 2 shows that even when all covariates were accounted for, increasing duration of relationship remained significantly associated with declining rates of all of the mental health problem measures $(P<0.05)$, with the exception of anxiety disorders $(P>0.79)$.

In order to test for the presence of significant non-linear components in the adjusted associations between relationship duration and mental health, the models described above were modified so that relationship duration was represented by a series of three design variates (see Method for details). This analysis did not reveal any significant departures from linearity.

\section{Legal status of relationship}

The fitted model in Table 2 provides an opportunity to examine whether the legal status of a relationship is significantly associated with mental health outcomes after due allowance has been made for relationship duration and other covariates. In order to achieve this, the fitted model was extended to include two design variates representing the legal status of the relationship. The adjusted regression coefficients and standard errors for these variables are reported in Table 2. In all cases the relationship status variables were not significantly associated with the mental health outcomes, with the exception of depression, where the de facto relationship variable was statistically significant $(P<0.05)$. However, this significant result could be due to chance as a result of multiple tests of significance. To address this, a Bonferroni adjusted ${ }^{27}$ $P$-value $(P<0.004)$ was used to correct for multiple $(n=12)$ tests of significance. The de facto relationship variable was not statistically significant using this adjusted $P$-value. These findings imply that it is the duration of a partner relationship rather than its legal status that influences mental health outcomes.

\section{Gender differences}

Table 3 shows the results of a nested regression model in which relationship duration was nested within gender. The model was otherwise the same as the population-averaged GEE model described above. The table compares the adjusted regression coefficients for relationship duration for men and women. It also reports the $P$-value from a paired comparison of the adjusted coefficients for men and women. The table shows that for most of the mental health measures the coefficients for men and women were not significantly different (depression, anxiety disorders, suicidal ideation/attempt, total number of mental health problems, $P>0.19$ ). However, there were significant gender differences for the substance use disorder measures (alcohol abuse/dependence, $P<0.001$, and illicit drug abuse/dependence, $P<0.05)$. Inspection of the regression coefficients showed that the protective effect of relationship duration on substance use disorders was greater for women than for men.

\section{Discussion}

This paper examined the associations between duration of partnership and mental disorder using data from a 30 -year longitudinal study of a birth cohort of individuals. The results suggested that:

(a) increasing duration of relationship was associated with declining rates of mental disorder;

(b) the association persisted after due allowance was made for covariate factors;

(c) when duration of relationship was taken into account, the legal status of the relationship (legally or de facto married) was unrelated to mental disorder;

(d) increasing duration of relationship had greater benefits for reducing alcohol and illicit drug abuse/dependence among women than it did among men.

These findings suggest that partner relationships are protective for mental health, with the protective effect

Table 3 Comparison of covariate-adjusted associations between relationship duration and mental disorder for men and women

\begin{tabular}{|c|c|c|c|c|c|}
\hline & \multicolumn{2}{|c|}{ Men } & \multicolumn{2}{|c|}{ Women } & \multirow{2}{*}{$\begin{array}{l}P \text { for equality } \\
\text { of gender coefficients }\end{array}$} \\
\hline & $B$ & s.e. & $B$ & s.e. & \\
\hline Depression & -0.25 & 0.09 & -0.17 & 0.06 & $>0.43$ \\
\hline Anxiety disorder & -0.11 & 0.11 & 0.02 & 0.07 & $>0.19$ \\
\hline Suicidal ideation/attempt & -0.19 & 0.14 & -0.32 & 0.12 & $>0.43$ \\
\hline Alcohol abuse/dependence & -0.19 & 0.08 & -0.60 & 0.12 & $<0.001$ \\
\hline Illicit drug abuse/dependence & -0.27 & 0.13 & -0.72 & 0.18 & $<0.02$ \\
\hline Number of mental health problems & -0.15 & 0.05 & -0.21 & 0.04 & $>0.31$ \\
\hline
\end{tabular}


increasing as the duration of the relationship increases. Previous studies have reported that married individuals have lower rates of mental disorder than unmarried individuals, and that this difference may be due to several factors, including increased social and emotional support and greater wealth (for review see Waite ${ }^{28}$ ). It is possible that the associations between relationship duration and mental disorder reported in our paper may be explained by similar factors. For example, emotional support and financial stability may continue to increase over the course of a relationship and may in part account for the greater protective effects of longer-duration relationships.

The associations between relationship duration and mental disorder in the current study remained even after extensive adjustment for covariate factors that included measures of prior mental disorder. After adjustment, relationship duration was significantly associated with all of the mental disorders except for anxiety. The lack of association with anxiety disorders is puzzling and has no apparent explanation. However, the associations between relationship duration and the other mental health problems are consistent with previous research, which has indicated that adjusting for prior mental disorder and other covariate factors reduces, but does not eliminate, the associations between marriage and mental disorder. These findings suggest that the association between relationship duration and mental disorder is not due to the selection of psychologically healthy individuals into marriage and may instead reflect a causal relationship, with relationship duration having a protective effect on mental health. However, it remains possible that the associations observed in the current study may be due to covariate factors that were not controlled for. Although efforts were made to control for a wide range of covariates, it remains possible that there are additional covariate factors that were not included in the models and could explain the associations between relationship duration and mental disorder.

The finding that the legal status of the relationship (legally or de facto married) was not significantly associated with mental health problems contrasts with previous studies, which have reported that the decrease in rates of mental health problems is greater for those in legal marriages than those in de facto relationships. ${ }^{2,7,9}$ However, many of these studies did not control for relationship duration. The results of the current study suggest that it is possible that the greater protective effect experienced by those who are legally married may be due to the longer relationship duration in marriage relationships compared with de facto relationships, rather than the legal status of the relationship.

The findings of this study also suggested that, in some cases, the effects of relationship duration on mental health were different for men and women. Specifically, the protective effect of relationship duration on alcohol and illicit drug abuse/dependence was greater for women than for men. For all other outcomes, the protective effect of relationship duration was similar for both genders. These findings are consistent with studies that have reported that the protective effect of marriage on substance use disorders is greater for women. ${ }^{4,6}$ However, the finding that the effect of relationship duration on depression was similar for men and women contrasts with previous studies that have reported that the effect is greater for men. ${ }^{4,8}$

Although the findings of this study suggested that increasing duration of partner relationships is associated with decreasing rates of mental health problems, they did not address the question of whether individuals in long-term relationships may experience more rapid recovery from mental illness and lower rates of relapse than those in shorter relationships.

\section{Implications}

The finding that increasing relationship duration is associated with lower rates of mental health problems suggests that individuals who are identified as high risk for developing mental health problems may benefit from efforts to improve the stability and duration of their partner relationships, such as improved access to relationship counselling services.

\section{Limitations}

A limitation of this study is that the data relate to a birth cohort of individuals born in a particular place during a narrow time period. In particular, the cohort were born in New Zealand, where non-marriage cohabiting relationships are common. ${ }^{29}$ It is not clear to what extent the results of the current study will generalise to different cohorts of individuals born in different places and different time periods, especially individuals from societies in which non-marriage cohabiting relationships are less widespread.

This limitation notwithstanding, the results of this study suggest that being in a cohabiting relationship, regardless of whether the relationship is a legal or a de facto marriage, is associated with lower rates of mental disorder, with increasing duration of relationship being associated with declining rates of mental disorder.

Sheree J. Gibb, PhD, David M. Fergusson, PhD, L. John Horwood, MSC,

Christchurch Health and Development Study, University of Otago, Christchurch School of Medicine and Health Sciences, Christchurch, New Zealand

Correspondence: Sheree Gibb, Christchurch Health and Development Study, University of Otago, Christchurch, PO Box 4345, Christchurch, New Zealand. Email: sheree.gibb@otago.ac.nz

First received 9 Jun 2010, final revision 30 Sep 2010, accepted 19 Oct 2010

\section{Funding}

This research was funded by grants from the Health Research Council of New Zealand, the National Child Health Research Foundation, the Canterbury Medical Research Foundation and the New Zealand Lottery Grants Board. S.J.G. was supported by a University of Otago Division of Health Sciences Career Development Postdoctoral Fellowship.

\section{Acknowledgements}

S.J.G. had full access to all of the data in the study and takes responsibility for the integrity of the data and the accuracy of the data analysis.

\section{References}

1 Williams K. Has the future of marriage arrived? A contemporary examination of gender, marriage, and psychological well-being. J Health Soc Behav 2003; 44: 470-87.

2 Stack S, Eshleman JR. Marital status and happiness: a 17-nation study J Marriage Fam 1998; 60: 527-36.

3 Simon RW. Revisiting the relationships among gender, marital status, and mental health. Am J Sociol 2002; 4: 1065-96.

4 Horwitz AV, White HR, Howell-White S. Becoming married and mental health: a longitudinal study of a cohort of young adults. J Marriage Fam 1996; 58: 895-907.

5 Lamb KA, Lee GR, DeMaris A. Union formation and depression: selection and relationship effects. J Marriage Fam 2003; 65: 953-62.

6 Scott KM, Wells JE, Angermeyer M, Brugha TS, Bromet TS, Demyttenaere K, et al. Gender and the relationship between marital status and first onset of mood, anxiety and substance use disorders. Psychol Med 2010; 40 1495-505.

7 Brown SL. The effect of union type on psychological well-being: depression among cohabitors versus marrieds. J Health Soc Behav 2000; 41: 241-55.

8 Gove WR. The relationship between sex roles, marital status, and mental illness. Soc Forces 1972; 51: 34-44. 
9 Kim HK, McKenry PC. The relationship between marriage and psychological well-being: a longitudinal analysis. J Fam Issues 2002; 23: 885-911.

10 Fergusson DM, Horwood LJ. The Christchurch Health and Development Study: review of findings on child and adolescent mental health. Aust N Z J Psychiatry 2001; 35: 287-96.

11 Fergusson DM, Horwood L, Shannon FT, Lawton JM. The Christchurch Child Development Study: a review of epidemiological findings. Paediatr Perinat Epidemiol 1989; 3: 278-301.

12 World Health Organization. Composite International Diagnostic Interview (CIDI). WHO, 1993.

13 American Psychiatric Association. Diagnostic and Statistical Manual of Mental Disorders (4th edn) (DSM-IV). APA, 1994.

14 Fergusson DM, Horwood LJ, Lynskey MT. Childhood sexual abuse and psychiatric disorder in young adulthood. II. Psychiatric outcomes of childhood sexual abuse. J Am Acad Child Adolesc Psychiatry 1996; 35 : 1365-74.

15 Reid NA, Jackson PF, Gilmore A, Croft C. Test of Scholastic Abilities. Ministry of Education, 1981.

16 Elley WB, Irving JC. Revised socio-economic index for New Zealand. New Zeal J Educ Stud 1976; 11: 25-36.

17 Fergusson DM, Horwood LJ, Lynskey MT. A longitudinal study of early childhood education and subsequent academic achievement. Aust Psychologist 1994; 29: 110-5.

18 Wechsler D. Wechsler Intelligence Scale for Children - Revised. The Psychological Corporation, 1974.
19 Quay HC, Peterson DR. Manual for the Revised Behavior Problem Checklist. H.C. Quay \& D.R. Peterson, 1987.

20 Eysenck HM, Eysenck SBG. Manual of the Eysenck Personality Inventory London University Press, 1964.

21 Cloninger CRA. Systematic method for clinical description and classification of personality variants. Arch Gen Psychiatry 1987; 44: 573-88.

22 Woodward L, Fergusson DM, Horwood LJ. Effects of single-sex and coeducational secondary schooling on children's academic achievement Aust J Educ 1999; 43: 142-56.

23 Fergusson DM, Boden JM, Horwood LJ. Exposure to childhood sexual and physical abuse and adjustment in early adulthood. Child Abuse Negl 2008; 32: $607-19$

24 Liang KY, Zeger SL. Longitudinal data analysis using generalized linear models. Biometrika 1986; 73: 13-22.

25 Zeger SL, Liang KY. Longitudinal data analysis for discrete and continuous outcomes. Biometrics 1986; 42: 121-30.

26 Lee J. Covariance adjustment of rates based on the multiple logistic regression model. J Chronic Dis 1981; 34: 415-26.

27 Grove WM, Andreasen NC. Simultaneous tests of many hypotheses in exploratory research. J Nerv Ment Dis 1982; 170: 3-8.

28 Waite LJ. Does marriage matter? Demography 1995; 32: 483-507.

29 Statistics New Zealand. Social marital status for the census usually resident population count aged 15 years and over, 2006. Statistics New Zealand, 2010 (http://www.stats.govt.nz/Census/2006CensusHomePage/classificationcounts-tables/about-people/social-marital-status.aspx).

\section{First fictional report of folie à deux}

\section{Daniel Martins de Barros, Geraldo Busatto Filho}

The induced psychosis, also known as folie à deux, was first described in 1887 by Lasegue \& Falret and is characterised by the transmission of delusional beliefs from a psychotic individual to a healthy individual. The article described the disorder as a syndrome occurring predominantly in women living in sheltered, isolated conditions. The syndrome was characterised by the emergence of the same psychotic symptoms in members of a family while living together, or in two very close individuals, and the transmission of psychotic symptoms from an affected individual to one or more healthy persons.

However, Machado de Assis, the Brazilian author, wrote a fictional story published 8 years before the article by Lasegue \& Falret, which contains an accurate description of folie à deux. In his story, Rafael the Angel, Assis narrates the life of a man who believed that he was the angel Rafael himself. This man lived in isolation on a farm with his daughter, who had no contact with the outside world until the age of 15 . Shortly before his death her father found her a suitor, who soon realises she had been contaminated by her father's delusions: not only did she believe that he was an angel, but defended this notion despite the disbelief of her new-found fiancé. Following the death of her father she moved from the countryside to the city and 3 months later presented no delusional beliefs.

Assis' narrative presents a picture which combines all the elements subsequently described by Lasegue \& Falret: a woman living in isolation and closely related to a psychotic family member who manifested identical psychotic symptoms and was the influence and the primary cause of her delusion. Assis went further and described the therapeutic effect of separating the individuals.

This story illustrates that, above all, literature can contribute to psychiatry by offering descriptions of conditions in remarkable detail. As noted by Sigmund Freud in Writings on Art and Literature, 'the poetic treatment of a psychiatric theme can turn out to be correct without any sacrifice of its beauty', what can be verified in this 'first case report' of folie à deux.

Daniel Martins de Barros, Institute of Psychiatry, Geraldo Busatto Filho, Institute and Department of Psychiatry, Medicine School, University of Sao Paulo. 